\title{
Metaspin and dirishonic dark matter
}

\author{
Alfons J. Buchmann and Michael L. Schmid \\ Institut für Theoretische Physik, Universität Tübingen, \\ Auf der Morgenstelle 14, D-72076 Tübingen, Germany*
}

\begin{abstract}
The antisymmetry requirement of rishon bound state wave functions suggests a new rishon quantum number called $M$ spin. From $M$ spin conservation and the Nussinov-Weingarten-Witten theorem we predict the existence of a stable pseudoscalar dirishonic meson $\zeta$ that is lighter than the lightest neutrino. Its mass is estimated as $m_{\zeta}=10^{-9} \mathrm{eV}$. This particle could make up the major part of cold dark matter in the Universe.
\end{abstract}

PACS numbers: 12.60.Rc, 12.60.-i, 12.10.-g

\section{INTRODUCTION}

Among the various approaches to lepton and quark substructure [1], the rishon model, which has only two massless spin $1 / 2$ building blocks $T$ and $V$ interacting via color and hypercolor forces, stands out because of its simplicity. The rishon model [26 ] provides answers to fundamental questions that remain unanswered within the standard model. For example, it explains why color triplet quarks have fractional whereas color singlet leptons have integer charges with $|Q| \leq 1$, and why the electroweak anomalies of leptons and quarks cancel [6]. Furthermore, it explains the origin of weak interactions as a residual force on the rishon bound state level $[6,7]$, and suggests an explanation of the generation number [8].

On the other hand, a major problem has not been sufficiently clarified. This concerns the requirement that preon bound state wave functions must be antisymmetric under the interchange of two spin $1 / 2$ preons (Pauli exclusion principle). Originally, it was proposed that the overall antisymmetry of rishon bound states lies in the space-time degrees of freedom [3], specifically, that three massless rishons in the fundamental $(1 / 2,0+0,1 / 2)$ representation of the relativistic space-time group $S L(2, C)$ combine to a totally antisymmetric product state. Another approach employs quaternions, a noncommutative generalization of complex numbers connected with four spatial dimensions, and quaternionic quantum mechanics [9].

The Pauli exclusion principle is a general quantum mechanical concept based on the indistinguishability of identical particles and the permutation group $\mathcal{S}_{n}$, and the problem of constructing fully antisymmetric rishon bound states may be formulated in a still different manner. The problem can be most

*Electronic address: alfons.buchmann@uni-tuebingen.de; Electronic address: micha.1.schmid@gmx.net

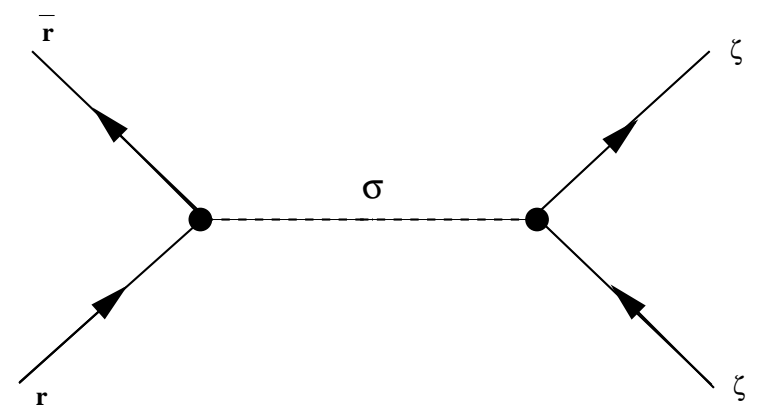

FIG. 1: Formation of a heavy scalaron $\sigma$ with mass $m_{\sigma}>$ $m_{G}=10^{16} \mathrm{GeV}$ from a rishon-antirishon pair $r \bar{r}$ and its decay into scalarinos $\zeta$.

clearly seen in the case of the color and hypercolor singlet positron $e^{+}(T T T)$, which consists of three $T$ rishons. This is studied in more detail in Sec. II, where we show that the Pauli principle can be satisfied if rishons carry an additional conserved SU(2) like quantum number, called metaspin $M$. The possible origin of metaspin is discussed in Sec. V.

As an interesting consequence of metaspin conservation we find in combination with the NussinovWeingarten-Witten (NWW) theorem [10] an argument for the existence of a stable dirishonic scalar bound state, which could be a candidate for cold dark matter. The argument goes like this. The NWW theorem applied to rishons requires a dirishonic scalar bound state that is lighter than the lightest three-rishon fermionic bound state, i.e. the neutrino. We call this particle scalarino $\zeta$. As a color and hypercolor singlet with spin $S=0$ and electric charge $Q=0$, the scalarino does not interact with ordinary matter via electromagnetic, weak or strong forces but only via short-range scalar interactions and gravitation. Scalarinos may have been produced via primordial rishon-antirishon annihilation into a heavy scalar boson, called scalaron $\sigma$, which subsequently decays into two scalarinos as depicted in Fig. 1. In Sec. III we argue that the scalaron must have a mass above the grand unified theory (GUT) scale of $10^{16} \mathrm{GeV}$ to prevent fermionic ris- 
hon bound states from having long-range scalarino mediated interactions, which are not observed. Furthermore, we will see that scalarinos have metaspin $M=1$. Therefore, they cannot decay into massless gauge bosons with metaspin $M=0$. This explains their stability once they have been formed. Possible consequences for cosmology and particle physics are studied in Sec. IV and Sec. VI.

\section{RISHON BOUND STATES, PAULI PRINCIPLE, AND METASPIN}

\section{A. Statement of the problem}

Rishons are spin $1 / 2$ particles. Therefore, according to the Pauli exclusion principle, rishon bound states must be fully antisymmetric under the interchange of two rishons of the same type. The consequences of this requirement are most transparent for the positron $e^{+}$, the rishon content of which is TTT. The positron is color and hypercolor neutral. As a result, its color-hypercolor product state is necessarily fully symmetric with respect to permutation of the rishon constituents.

We assume that the $e^{+}$orbital ground state is totally symmetric under permutation. Because the spin wave function of the $e^{+}$is mixed symmetric, there must be another degree of freedom in which the three $T$ rishons are in a state of mixed symmetry to generate an overall totally antisymmetric wave function for the positron. This additional degree of freedom cannot be $\mathrm{SU}(2)$ isospin as in the quark model. For $T$ and $V$ rishons there is no global $\mathrm{SU}(2)$ isospin symmetry because they are in different color representations [3] as is evident from Table I. Thus, we postulate that rishons carry a new SU(2) quantum number, called metaspin $M$. Although the above argument requires a noncovariant separation of spin and space coordinates, it is not in conflict with special relativity. For particles with nonzero rest mass, it is possible to define orbital and spin symmetries separately [11].

The Pauli principle demands that the product wave function of rishon bound states $|B\rangle$

$$
|B\rangle=|O\rangle \times|H\rangle \times|C\rangle \times|S\rangle \times|M\rangle,
$$

must be a fully antisymmetric representation of the direct product group of orbital $(\mathrm{O})$, hypercolor $(\mathrm{H})$, color $(\mathrm{C})$, spin $(\mathrm{S})$ and metaspin $(\mathrm{M})$ groups

$$
O(3)_{O} \times S U(3)_{H} \times S U(3)_{C} \times S U(2)_{S} \times S U(2)_{M} .
$$

Individual $T$ and $V$ rishons are represented by Young diagrams according to the quantum number assignments of Table I as

$$
|T\rangle=\square O \times \square{ }_{H} \times \square{ }_{C} \times \square_{S} \times \square_{M}
$$

\begin{tabular}{lcccccccc}
\hline Rishon & $\mathrm{H}$ & $\mathrm{C}$ & $\mathrm{Q}$ & $\mathcal{P}$ & $\Upsilon$ & $\Pi$ & $\mathrm{S}$ & $\mathrm{M}$ \\
\hline$T$ & 3 & 3 & $+\frac{1}{3}$ & $+\frac{1}{3}$ & $+\frac{1}{3}$ & -1 & $\frac{1}{2}$ & $\frac{1}{2}$ \\
\hline$V$ & 3 & $\overline{3}$ & 0 & $+\frac{1}{3}$ & $-\frac{1}{3}$ & +1 & $\frac{1}{2}$ & $\frac{1}{2}$ \\
\hline $\bar{V}$ & $\overline{3}$ & 3 & 0 & $-\frac{1}{3}$ & $+\frac{1}{3}$ & -1 & $\frac{1}{2}$ & $\frac{1}{2}$ \\
\hline $\bar{T}$ & $\overline{3}$ & $\overline{3}$ & $-\frac{1}{3}$ & $-\frac{1}{3}$ & $-\frac{1}{3}$ & +1 & $\frac{1}{2}$ & $\frac{1}{2}$ \\
\hline
\end{tabular}

TABLE I: The hypercolor $(\mathrm{H})$, color $(\mathrm{C})$, electric charge $(\mathrm{Q})$, rishon number $(\mathcal{P}), \Upsilon$ number $(\Upsilon)$, intrinsic parity $(\Pi)$, spin (S), and metaspin (M) of rishons and antirishons. The quantum numbers $\mathcal{P}$ and $\Upsilon$ are defined as $\mathcal{P}=(n(T)+n(V)) / 3$ and $\Upsilon=(n(T)-n(V)) / 3$, where $n(T)$ and $n(V)$ are the number of $T$ and $V$ rishons, and $Q=(\mathcal{P}+\Upsilon) / 2$

$$
|V\rangle=\square{ }_{O} \times \square_{H} \times \square_{C} \times \square_{S} \times \square_{M}
$$

noting that the $V$ rishon is an $\mathrm{SU}(3)_{C}$ antitriplet $\mathbf{3}_{C}$ (see Table I) represented by two boxes just as an antisymmetric two-rishon state.

We then form outer products of multi-rishon states in orbital, color, hypercolor, spin, and metaspin space separately. In the following, we discuss these outer products for fermions (leptons, quarks, and hyperquarks [12]) and spin 1 bosons (dirishons and six-rishons). Dirishonic bound states with spin 0 are discussed in Sec. III and Sec. IV.

\section{B. Leptons, quarks, and hyperquarks}

We begin with the orbital wave function. For the three-rishon bound states listed in Table II and their antiparticles, we assume that the $\mathrm{O}(3)_{O}$ orbital wave function is the fully symmetric Young tableau

Next, we construct the outer product of three rishons in hypercolor and color spaces separately, and in a second step, form hypercolor-color inner product states. For the hypercolor and color singlet leptons containing three preons of the same type (see Table II) one obtains, e.g., for the positron $e^{+}(T T T)$

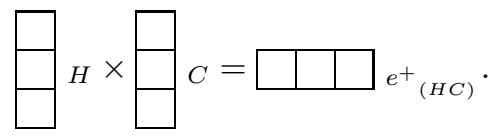

Analogous results are obtained for the neutrino, so that leptons have fully symmetric hypercolor-color product states.

For quarks, the hypercolor product state is again the fully antisymmetric $S U(3)_{H}$ singlet, whereas in color space a color triplet is required. For example, for a $u(T T V)$ quark, the outer product (denoted by $\otimes)$ of the two color-triplet $T$ and the color-antitriplet $V$ rishons leads to the following irreducible $S U(3)_{C}$ representations with color dimensions as subscripts: 


\begin{tabular}{|c|c|c|c|c|c|c|c|}
\hline & Rishon content & Bound state & $\mathrm{H} \mathrm{C}$ & $\mathcal{P}$ & $\Upsilon$ & $Q$ & $\mathrm{~S} \mathrm{M}$ \\
\hline & $(V V V)$ & $\left(\nu_{e}, \nu_{\mu}, \nu_{\tau}\right)$ & 11 & +1 & -1 & 0 & $\frac{1}{2} \quad \frac{1}{2}$ \\
\hline \multicolumn{8}{|l|}{ Leptons } \\
\hline & $(\bar{T} \bar{T} \bar{T})$ & $\left(e^{-}, \mu^{-}, \tau^{-}\right)$ & 11 & -1 & -1 & -1 & $\frac{1}{2} \quad \frac{1}{2}$ \\
\hline & $(T T V)$ & $(u, c, t)$ & 13 & +1 & $+\frac{1}{3}$ & $+\frac{2}{3}$ & $\frac{1}{2} \quad \frac{1}{2}$ \\
\hline \multicolumn{8}{|l|}{ Quarks } \\
\hline & $(\bar{T} \bar{V} \bar{V})$ & $(d, s, b)$ & 13 & -1 & $+\frac{1}{3}$ & $-\frac{1}{3}$ & $\frac{1}{2} \quad \frac{1}{2}$ \\
\hline \multirow{3}{*}{ Hyperquarks } & $(T T \bar{V})$ & $(\tilde{u}, \tilde{c}, \tilde{t})$ & $\begin{array}{ll}3 & 1\end{array}$ & $+\frac{1}{3}$ & +1 & $+\frac{2}{3}$ & $\frac{1}{2} \quad \frac{1}{2}$ \\
\hline & & & & & & & \\
\hline & $(\bar{T} V V)$ & $(\tilde{d}, \tilde{s}, \tilde{b})$ & $\begin{array}{ll}3 & 1\end{array}$ & $+\frac{1}{3}$ & -1 & $-\frac{1}{3}$ & $\frac{1}{2} \quad \frac{1}{2}$ \\
\hline
\end{tabular}

TABLE II: Allowed three-rishon bound states representing leptons, quarks and hyperquarks and their quantum numbers. Formally, the hyperquarks are obtained from the corresponding quarks by interchanging: $V \leftrightarrow \bar{V}$.

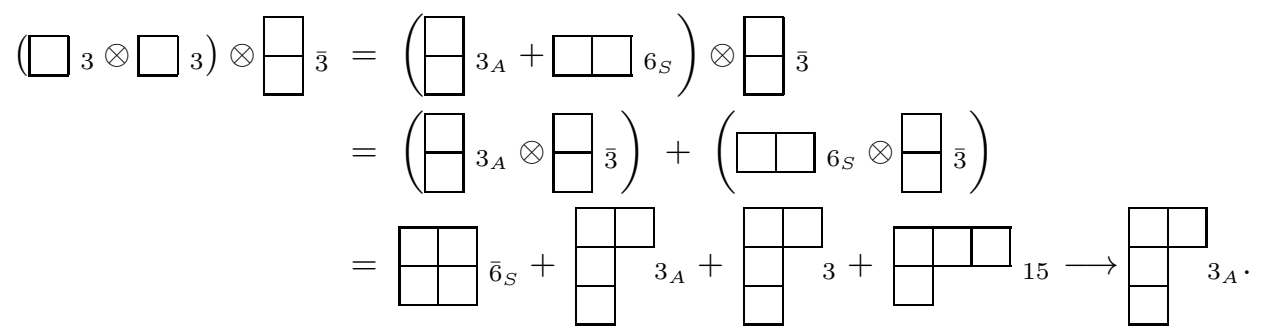

The same irreducible representations are obtained if one first combines the color-triplet $T$ with the colorantitriplet $V$. Because the latter is distinguishable from the former, we need only antisymmetrize the $T$ rishons. This eliminates the diagrams arising from the symmetric $(T T)_{6_{S}}$. From the remaining two diagrams generated by the coupling of the antisymmetric $(T T)_{3_{A}}$ and $(V)_{\overline{3}}$ Young schemes, only the antisymmetric color triplet $3_{A}$ (indicated by the arrow) has the correct total color symmetry. While Eq.(6) contains the proper $S U(3)_{C}$ dimension of the $u$-quark, with respect to the permutation group $\mathcal{S}_{3}$, we have only three rishons and the permutational symmetry in hypercolor-color space is represented by three boxes in a single row as on the right-hand side of Eq.(5).

For leptons and quarks, our results concerning the permutational symmetry of the combined hypercolor-color product states are consistent with those of Harari and Seiberg [3]. For hyperquarks, e.g., $\tilde{u}(T T \bar{V})$ the role of color and hypercolor is interchanged, otherwise the construction of hypercolorcolor wave functions is analogous to that of quarks. Thus, for leptons, quarks, and hyperquarks, the inner product of hypercolor and color spaces is the completely symmetric representation conjugate to the fully antisymmetric spin-metaspin product representation constructed next.
From three rishons with spin $1 / 2$ and metaspin $1 / 2$ we first construct mixed symmetric outer product states in spin and metaspin space separately. These mixed symmetric spin and metaspin parts are then combined to form a fully antisymmetric inner product wave function common to all fermionic bound states. In principle, two mixed symmetric states can combine to fully symmetric, mixed symmetric, and fully antisymmetric $\mathcal{S}_{3}$ product states

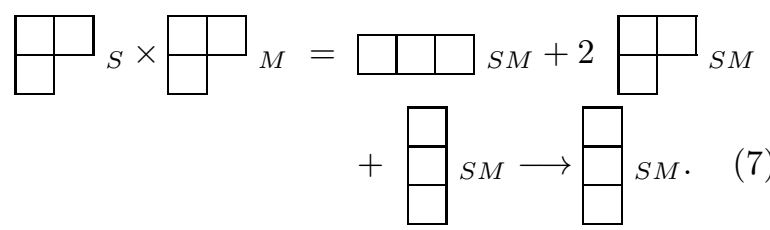

As indicated by the arrow we confine ourselves to the totally antisymmetric product state. The reason for this choice is particularly evident for the positron, where the orbital-color-hypercolor product state totally symmetric (see above). As a result, the antisymmetry of fermionic rishon bound states lies in the combined spin-metaspin space.

The overall product states of fermionic rishon bound states are then

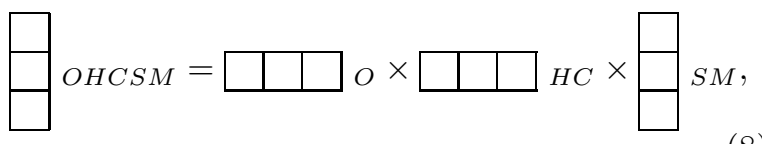


i.e. totally antisymmetric with respect to permutations of the rishon constituents. Thus, the metaspin hypothesis allows us to construct fully antisymmetric three-rishon bound states.

In addition, metaspin explains why there are no leptons, quarks, and hyperquarks with spin $S=3 / 2$. Spin $3 / 2$ states are totally symmetric, which requires fully antisymmetric metaspin wave functions. However, this is impossible in $\mathrm{SU}(2)_{M}$, where the maximum number of rows in any Young scheme is restricted to two. Interestingly, Harari and Seiberg [3] could also exclude spin $3 / 2$ lepton states due to the antisymmetry of their relativistic space-time wave function but needed an additional argument to exclude spin $3 / 2$ quarks.

\section{Dirishons with spin $\mathrm{S}=1$}

The dirishons $N(V V), U(T \bar{V})$, and $\tilde{U}(T V)$ with spin $S=1$ have been discussed in detail in Ref. [7], where we have shown that the neutralons $N(V V)$ transform hyperquarks into quarks, while the $U$ and $\tilde{U}$ bosons are responsible for transitions between leptons and quarks and between leptons and hyperquarks respectively. Hence, it suffices to reproduce in Table III the quantum numbers of these SU(9) gauge bosons.

As in the case of the three-rishon bound states, the hypercolor-color product state of dirishons is fully symmetric. Because dirishons with spin $S=1$ are

\begin{tabular}{lccccc}
\hline Dirishon & $\mathrm{H}$ & $\mathrm{C}$ & $\mathcal{P}$ & $\Upsilon$ & $Q$ \\
\hline$N(V V)$ & $\overline{3}$ & 3 & $+\frac{2}{3}$ & $-\frac{2}{3}$ & 0 \\
\hline $\bar{N}(\bar{V} \bar{V})$ & 3 & $\overline{3}$ & $-\frac{2}{3}$ & $+\frac{2}{3}$ & 0 \\
\hline \hline$U(T \bar{V})$ & 1 & $\overline{3}$ & 0 & $+\frac{2}{3}$ & $+\frac{1}{3}$ \\
\hline $\bar{U}(\bar{T} V)$ & 1 & 3 & 0 & $-\frac{2}{3}$ & $-\frac{1}{3}$ \\
\hline$\tilde{U}(T V)$ & $\overline{3}$ & 1 & $+\frac{2}{3}$ & 0 & $+\frac{1}{3}$ \\
\hline$\tilde{U}(\bar{T} \bar{V})$ & 3 & 1 & $-\frac{2}{3}$ & 0 & $-\frac{1}{3}$ \\
\hline
\end{tabular}

TABLE III: Quantum numbers of dirishonic bound states with $S=1$ and $M=0$.

in a fully symmetric representation in spin space, they must be in a fully antisymmetric representation in metaspin space corresponding to $M=0$ to form completely antisymmetric spin-metaspin inner product states

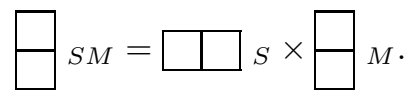

The total wave function of dirishons with $S=1$ is then fully antisymmetric

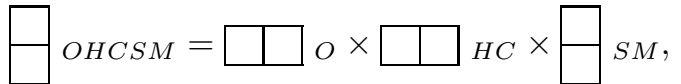

as in the case of fermionic rishon bound states.

\section{Six-rishon bound states with $\operatorname{spin} S=1$}

Previously [7], we have seen that there are transitions between dirishons and six-rishon bound states with $S=1$. For example, in hyperbaryon decay, three neutralons $N(V V)$ can combine to a $\chi$-boson

$$
3 N(V V) \longrightarrow \chi\left(\begin{array}{c}
V V V \\
V V V
\end{array}\right) .
$$

Since neutralons have metaspin $M=0$, it follows that the $\chi$-boson has metaspin $M=0$ as well. Furthermore, as a result of neutrino-antineutrino oscillations [7], the $\chi$ can oscillate into the weak $Z$-boson and the $\bar{\chi}$

$$
\chi \longleftrightarrow Z \longleftrightarrow \bar{\chi}
$$

Because the $\chi, Z$, and other six-rishons with spin $S=1$, as well as the dirishons in Table III belong to the same supermultiplet of the effective $\mathrm{SU}(9)$ gauge group [7], we conclude that all spin 1 gauge bosons, in particular photons, gluons, and hypergluons have metaspin $M=0$. This has consequences for the stability of the scalar dirishons to be discussed in the next section.

\section{NWW THEOREM AND THE LIGHTEST DIRISHON}

The Nussinov-Weingarten-Witten theorem [10], which is valid in a QCD like theory, provides an inequality between baryon and meson masses. It has been proven using n-flavor lattice QCD techniques by Weingarten and independently by Nussinov using gluon exchange dynamics and the variational principle. In QCD, the theorem states that there is a colorless pseudoscalar quark-antiquark bound state which is lighter than the lightest colorless threequark bound state. More specifically, this leads to the following inequality [10]

$$
m_{\pi} \leq \frac{2}{3} m_{N},
$$

where $m_{\pi}$ and $m_{N}$ are the pseudoscalar pion and nucleon masses.

In QCD these inequalities appear to be connected with spontaneous chiral symmetry breaking $\mathrm{S} \chi \mathrm{SB}$. However, in the present vectorlike theory, chiral symmetry is preserved as required by 't Hooft anomaly conditions. As shown in his original paper, 't Hooft's anomaly matching condition [13] can be satisfied in a vectorlike theory with only 2 fundamental fermion flavors but not in QCD with 3 quark flavors. Therefore, in QCD with three (and more) 
quark flavors, chiral symmetry is necessarily spontaneously broken. This need not be the case in the present rishon model based on only 2 elementary ( $\mathrm{T}$ and $\mathrm{V}$ rishon) flavors. In this model, the presence of the $\mathrm{SU}(3)_{C}$ group and the possibility of obtaining a third class of fermionic bound states (hyperquarks) affect the anomaly matching conditions and change the pattern of chiral symmetry breaking compared to the one of a single hypercolor $\mathrm{SU}(3)_{H}$ group. Previously we have shown that for the global U(1) preon number and global B-L symmetries, 't Hooft anomaly matching may be satisfied if a new group of $\mathrm{SU}(3)_{C}$ color singlet fermionic bound states, called hyperquarks, is introduced [8]. Thus, there is some evidence that 't Hooft's anomaly matching conditions are not necessarily in conflict with vectorlike confining theories. It is therefore not unreasonable to expect that spontaneous chiral symmetry breaking can be avoided in the present model.

Below we argue that $\mathrm{S} \chi \mathrm{SB}$ is not a necessary condition for the validity of the NWW theorem. First, the NWW mass inequality $M_{B} \geq$ $(3 / 2) M_{M}$ is also valid for the zero mass case for both mesons and baryons thereby excluding both the Nambu-Goldstone (massless pseudoscalars, massive fermions) and the Wigner-Weyl (massive pseudoscalars, massless fermions) modes of chiral symmetry breaking. Second, baryon-meson mass inequalties can be derived using a simple one-gluon exchange potential model and the variational priniciple. In particular, in Nussinov's one-gluon exchange model derivation the relation $V_{q q}=2 / 3 V_{q \bar{q}}$ solely based on the evaluation of the color generators $\lambda_{1} \cdot \lambda_{2}$ for mesons and baryons is essential. This makes it clear that the validity of meson-baryon mass inequalities is not limited to theories which display S $\chi \mathrm{SB}$. The latter is a sufficient but not a necessary condition for the validity of the NWW theorem. This allows the existence of nearly massless pseudoscalar mesons which are not the Goldstone bosons connected with $\mathrm{S} \chi \mathrm{SB}$ and of nearly massless fermionic preonic bound states. In Ref. [14] it is clearly stated that $\mathrm{S} \chi \mathrm{SB}$ implies the existence of massless pseudoscalar particles but that the converse is not true, i.e. the existence of nearly massless pseudoscalar particles (e.g. the dirishonic $\zeta$ ) does not imply that chiral symmetry is spontaneously broken. As a result, the NWW theorem is valid in theories that do not display spontaneously broken chiral symmetry.

When applied to rishons, the theorem requires the existence of a hypercolor neutral, dirishonic, pseudoscalar bound state with a mass smaller than the lightest hypercolor neutral three-rishon bound state of the theory, i.e. the neutrino. The simplest hypercolor neutral, rishon-antirishon bound state is the pseudoscalar combination composed of $T \bar{T}$ and $V \bar{V}$

$$
\zeta=\frac{1}{\sqrt{2}}(T \bar{T}-V \bar{V}) .
$$

We call this particle scalarino $\zeta$. There is also an orthogonal state, called scalaron $\sigma$,

$$
\sigma=\frac{1}{\sqrt{2}}(T \bar{T}+V \bar{V}) .
$$

The scalarino $\zeta$ corresponds to the neutral pion $\left|\pi^{0}\right\rangle=(1 / \sqrt{2})|u \bar{u}-d \bar{d}\rangle$, and the scalaron $\sigma$ to the heavy scalar meson $\left|f^{0}\right\rangle=(1 / \sqrt{2})|u \bar{u}+d \bar{d}\rangle$ in QCD.

As color and hypercolor singlets, the $\zeta$ and $\sigma$ have fully symmetric hypercolor-color product wave functions. Because these spin 0 states are composed of distinguishable particles (rishons and antirishons) we cannot infer their metaspin quantum number from the requirement of antisymmetry of the total wave function. However, we have seen in Sec. II that dirishons with $S=1$, e.g., the $N(V V)$, must have metaspin $M=0$ for the spin-metaspin wave function to be completely antisymmetric. Likewise, for fermionic rishon bound states an antisymmetric spin-metaspin wave function was required. Extending this pattern to the scalar $\zeta$ and $\sigma$, these states must have $M=1$ (see Table IV). This assignment is supported by considering the charged scalar partners of the $\sigma$, which necessarily have $M=1$, and their metaspin conserving decays in Eq.(19).

Based on the NWW theorem, metaspin conservation, and the preon-triality rule, we deduce that scalarinos $\zeta$ have the following properties.

First, for the scalarino mass, the NWW theorem [10] implies

$$
m_{\zeta} \leq \frac{2}{3} m_{\nu_{e}}
$$

From our estimate for the neutrino mass $m_{\nu_{e}} \cong 10^{-8}$ $\mathrm{eV}$ [7] follows that $m_{\zeta} \leq 10^{-8} \mathrm{eV}$.

Second, because of metaspin conservation, the $\zeta$ with metaspin $M=1$ cannot decay into massless photons and gluons because the latter have metaspin $M=0$ as discussed in Sec. II.

Third, scalarinos do not interact with fermions below the GUT scale $M_{G}$. Otherwise, the small $\zeta$ mass would entail a long-range scalar interaction between fermions that is not observed. The suppression of fermion-scalarino interactions is guaranteed by the preon triality rule [7]. The latter states that below energies of order $M_{G}$ vacuum creation and annihilation of dirishonic bosons is forbidden; only a simultaneous vacuum creation and annihilation of 3 preon-antipreon pairs is allowed. Therefore, for $E \leq M_{G}=10^{16} \mathrm{GeV}$ one has the following constraint (preon triality rule)

$$
n_{(\bar{T} T)}+n_{(\bar{V} V)}=3 k
$$


where $n_{(\bar{T} T)}, n_{(\bar{V} V)}$, and $k$ are natural numbers. Thus, scalarinos have only been created above the GUT scale from rishon-antirishon annihilation into a heavy scalaron and its subsequent decay as depicted in Fig. 1, thereby violating Eq.(16). For the same reason there is no $\zeta \bar{\zeta}$ pair annihilation into two photons or production of $\zeta \bar{\zeta}$ pairs from two photons below the GUT scale.

The $\zeta$ mass can also be estimated from the relation $[15]$

$$
m_{\zeta}=\frac{1}{f_{G}} m_{\pi} f_{\pi}
$$

where $f_{\pi} \cong 93 \mathrm{MeV}$ is the pion decay constant and $m_{\pi} \cong 140 \mathrm{MeV}$ is the pion mass. Here, $f_{G}$ is the scale where a single rishon-antirishon pair can fuse via a preon-triality process into a dirishonic scalar $\sigma$ as in Fig. 1. Originally, Eq.(17) with $m_{\zeta}$ and $f_{G}$ replaced respectively by the axion mass $m_{A}$ and the Peccei-Quinn symmetry breaking scale $v$ was derived using current algebra techniques to obtain bounds on the mass and couplings of the invisible axion. Here, we obtain with $f_{G} \geq M_{G}=10^{16} \mathrm{GeV}$ a scalarino mass of $m_{\zeta} \leq 10^{-9} \mathrm{eV}$ in agreement with the bound obtained from the NWW theorem, Eq.(15). The mass of the dirishonic $\sigma$ boson must then be in the range $f_{G}<m_{\sigma} \leq 10^{17} \mathrm{GeV}$. Above $10^{18} \mathrm{GeV}$ no bound state can exist and rishons appear as asymptotically free particles [7].

In summary, the scalarino is the lightest rishon bound state, which is furthermore absolutely stable and inert.

\section{HEAVY DIRISHONIC SCALARS}

In this section we briefly comment on another interesting aspect of heavy dirishonic scalar bound states. The neutral scalaron $\sigma$ with metaspin $M=1$ provides an additional binding force between rishons with metaspin $1 / 2$ at a mass scale of $m_{\sigma} \cong 10^{17} \mathrm{GeV}$ described by the Lagrangian

$$
\mathcal{L}_{\sigma}=g_{M} \bar{\psi} \boldsymbol{\sigma}_{M} \cdot \phi_{\sigma} \psi
$$

where $g_{M}$ is the rishon-scalaron coupling constant, $\boldsymbol{\sigma}_{M}$ is the metaspin Pauli matrix, $\boldsymbol{\phi}_{\sigma}$ is the metaspin vector scalaron, and $\psi$ the rishon field.

The existence of a short-ranged attractive $\sigma$ exchange interaction explains why the dirishonic bosons $N(V V), U(T \bar{V})$, and $\tilde{U}(T V)$ with $S=1$ and $M=0$ (see Table III) are bound with a strength $g_{M}>g_{G}$, where $g_{G}=\sqrt{4 \pi \alpha_{G}}$ is the coupling strength of grand unification [7]. The binding of these colored and/or hypercolored gauge bosons cannot be fully explained by color and hypercolor $S U(3)$ forces and requires an additional short-range scalar attraction. Without this scalar binding the situation would be as in QCD where color unsaturated $q q$ states do not exist. The same scalar binding mechanism explains the relative stability of six-rishon bound states, e.g. the weak gauge bosons $W$ and $Z$ of the standard model and the gauge bosons of the $S U(6)_{P}$ and $S U(9)_{G}$ gauge groups [7] with saturated metaspin $M=0$.

The $\sigma$-binding force is also responsible for the formation of dirishonic $\sigma^{+}(T T)$ and $\sigma^{-}(\bar{T} \bar{T})$ bound states (see Table IV). Because these states do not occur in the $S U(9)_{G}$ gauge group, they must be spin scalars, and therefore have metaspin $M=1$ as discussed in Sec. III. In analogy to fermionic bound states, where $m_{e}>m_{\nu}$, the charged scalars have a larger mass than their neutral counterparts $m_{\sigma^{ \pm}} \geq m_{\sigma^{0}}$. Furthermore, because they participate in both the $\sigma$-interaction and in color-hypercolor interactions they are expected to have a larger decay width compared to the $\sigma^{0}$. Their prevalent metaspin conserving decay modes are

$$
\begin{array}{ll}
\sigma^{+} \longrightarrow & \sigma^{0}+U+\tilde{U} \\
\sigma^{-} \longrightarrow & \sigma^{0}+\bar{U}+\tilde{\tilde{U}}
\end{array}
$$

Here, metaspin $M=1$ is carried by the scalars $\sigma^{ \pm}$ and $\sigma^{0}$, whereas the vector bosons $U$ and $\tilde{U}$ have $M=0$ (see table III). This completes our discussion of the spectrum of dirishonic scalars.

\begin{tabular}{lcccccc}
\hline Dirishon & H C & $\mathcal{P}$ & $\Upsilon$ & $Q$ & $\Pi$ \\
\hline$\sigma^{+}(T T)$ & $\overline{3}$ & $\overline{3}$ & $+\frac{2}{3}$ & $+\frac{2}{3}$ & $+\frac{2}{3}$ & +1 \\
\hline$\sigma^{-}(\bar{T} \bar{T})$ & 3 & 3 & $-\frac{2}{3}$ & $-\frac{2}{3}$ & $-\frac{2}{3}$ & +1 \\
\hline$\sigma^{0}(T \bar{T}+V \bar{V}) / \sqrt{2}$ & 1 & 1 & 0 & 0 & 0 & +1 \\
\hline$\zeta(T \bar{T}-V \bar{V}) / \sqrt{2}$ & 1 & 1 & 0 & 0 & 0 & -1 \\
\hline
\end{tabular}

TABLE IV: Quantum numbers of scalar dirishonic bound states with $S=0$ and $M=1$.

\section{METASPIN AND A COMPACTIFIED FOURTH SPATIAL DIMENSION}

With the help of metaspin, we could construct fully antisymmetric rishon bound states, and together with the NWW theorem predict the existence of the lightest dirishonic bound state $\zeta$ but have avoided the question of the origin of this quantum number. At present we can only speculate that metaspin appears to be connected with an extended space-time symmetry involving a compactified fourth spatial dimension, as suggested by the isomorphism between $\mathrm{SU}(2)_{S} \times \mathrm{SU}(2)_{M} \sim \mathrm{SO}(4)$, rather than being an internal symmetry. 
This is reminiscent of Kaluza-Klein theory [16], which provides a common framework for gravitational and electromagnetic forces by extending the number of spatial dimensions to four. Kaluza-Klein theory supplies a length scale $l$ for the rolled-up fourth spatial dimension [17]

$$
l=\frac{\hbar \sqrt{16 \pi G}}{e c}=l_{P l} \frac{2}{\sqrt{\alpha_{Q}}} \sim 10^{-33} \mathrm{~m},
$$

where $G$ is Newton's gravitational constant, $l_{P l}=$ $\sqrt{\hbar G / c^{3}}$ is the Planck scale of quantum gravity and $\alpha_{Q}=e^{2} /(4 \pi \hbar c)$ the Sommerfeld constant. The length scale $l$ corresponds to an energy scale $E \sim$ $10^{17} \mathrm{GeV}$.

Interestingly, this is close to the preon scale $M_{p r} \sim$ $10^{18} \mathrm{GeV}$, discussed in Ref. [7]. It is also in the energy range $10^{17}-10^{18} \mathrm{GeV}$ where metaspin plays an important role in preonic interactions, in particular in the formation of rishonic bound states satisfying the Pauli principle. At the same energy scale, the metaspin conserving decays of the charged heavy scalars $\sigma^{ \pm}$into the $\mathrm{SU}(9)$ gauge bosons $U$ and $\tilde{U}$ and the scalaron $\sigma^{0}$ as in Eq.(19) occur. Furthermore, at around $10^{17} \mathrm{GeV}$ the latter decays into the light scalarinos as shown in Fig. 1. Therefore, the length scale where metaspin plays an important role coincides with the scale $l$ of the compactified fourth dimension according to Kaluza-Klein theory. This suggests that the $\mathrm{SU}(2)$ metaspin symmetry may be connected with the periodicity generated by rolling up the fourth spatial dimension.

After leptons, quarks, and hyperquarks have been formed, metaspin is still a conserved quantum number, but ceases to play an active role at larger length scales, e.g., in the antisymmetrization of three-quark bound states. Similarly, we need not antisymmetrize the quarks in one hydrogen atom with those in the other when construcing the wave function of the $\mathrm{H}_{2}$ molecule.

\section{DIRISHONIC COLD DARK MATTER}

The properties of the light pseudoscalar $\zeta$ discussed in Sec. III would make this particle an interesting cold dark matter candidate as outlined below. Because of the preon triality rule in Eq.(16), the $\zeta$ was produced only primordially at energies above $M_{G}$ via the decay of a heavy $\sigma$ boson with mass $m_{\sigma}>M_{G}$ as shown in Fig. 1 .

The large mass of the $\sigma$ implies that scalarinos were created before quarks and leptons were formed. On the other hand, due to their small mass, scalarinos may have been more copiously produced than any other rishonic bound state in the early Universe. An inflatory expansion near the GUT scale deceler- ated the scalarinos to velocities close to the nonrelativistic limit. Furthermore, the $\zeta$ has metaspin $M=1$ and thus cannot decay into massless photons and gluons with $M=0$ due to metaspin conservation. As a result, below the GUT scale, the $\zeta$ appears as an absolutely stable, inert, nearly massless particle.

What additional cosmological insights can be drawn from this scenario? Given the scalarino mass deduced from Eq.(15) and Eq.(17), we can estimate the number of scalarinos in the Universe following the arguments in Ref. [18]. It is known [19] that the ratio of baryonic density to the dark matter density of the Universe is approximately $\rho_{B} / \rho_{D M} \cong 0.2$. Assuming that scalarions are the only source of dark matter, i.e., $\rho_{D M}=\rho_{\zeta}$ we get for the corresponding matter densities the following expressions

$$
\begin{aligned}
\rho_{B} & =n_{B} m_{B}=\epsilon_{B} n_{\gamma} m_{B} \\
\rho_{\zeta} & =n_{\zeta} m_{\zeta},
\end{aligned}
$$

where $n_{B}, n_{\gamma}$, and $n_{\zeta}$ are the number densities of baryons, photons, and scalarinos. Furthermore, $m_{B} \cong 1 \mathrm{GeV}$ is the baryon mass and $\epsilon_{B}=n_{B} / n_{\gamma}=$ $610^{-10}$ is the baryon asymmetry [20]. We then get with $m_{\zeta} \cong 10^{-9} \mathrm{eV}$

$$
\frac{n_{\zeta}}{n_{\gamma}}=\frac{\rho_{\zeta}}{\rho_{B}} \frac{m_{B}}{m_{\zeta}} \epsilon_{B}=310^{9} .
$$

Therefore, the ratio of scalarino to photon number density is approximately equal to the ratio of photon to baryon number density, i.e., we have $10^{9}$ times more scalarinos than photons per volume. This suggests a very homogeneous distribution of dirishonic cold dark matter in the Universe.

\section{SUMMARY}

To satisfy the requirement of total antisymmetry of rishon bound state wave functions we have introduced an $\mathrm{SU}(2)$ like quantum number $M$, called metaspin. We could then construct totally antisymmetric fermionic and bosonic rishon bound states. In each case, the antisymmetry resides in the spinmetaspin product space. As a result, $S=1$ bosons necessarily have $M=0$, whereas $S=0$ bosons have $M=1$. Due to the isomorphism $\mathrm{SU}(2)_{S}$ $\times \mathrm{SU}(2)_{M} \sim \mathrm{SO}(4)$, it has been suggested that metaspin could be connected with the existence of a fourth spatial dimension, which is compactified and limited to distances near the Planck scale.

Based on the Nussinov-Weingarten-Witten theorem applied to the rishon model, we have deduced the existence of a pseudoscalar dirishonic meson, called scalarino $\zeta$, that is lighter than the lightest neutrino and hence is the lightest rishon bound 
state. We have estimated the scalarino mass to be of order $m_{\zeta} \cong 10^{-9} \mathrm{eV}$.

The stability, abundance, and inertness of scalarinos has been derived from the following arguments. First, due to $M$ spin conservation, scalarinos with $M=1$ cannot decay into massless gauge bosons with $M=0$. Second, scalarinos were created only at energies above the GUT scale of $M_{G} \cong 10^{16} \mathrm{GeV}$ with a production rate that vastly exceeds that of any other rishon bound state. We have estimated the ratio of cosmic scalarino and photon number densities as $n_{\zeta} / n_{\gamma} \sim 10^{9}$. Third, below the GUT scale, scalarinos interact with other particles only via grav- itational interaction and induced higher order electromagnetic interactions, which will make their detection difficult. Nevertheless, it appears that its properties make the scalarino a viable cold dark matter candidate.

\section{Acknowledgments}

We thank H. Harari, D. Lichtenberg, and I. Obukhovsky for useful discussions.
[1] I. A. D'Souza and C. S. Kalman, Preons (World Scientific, Singapore, 1992).

[2] H. Harari, Phys. Lett. 86B, 83 (1979).

[3] H. Harari, N. Seiberg, Phys. Lett. 98B, 269 (1981); Nucl. Phys. B204, 141 (1982).

[4] H. Harari, N. Seiberg, Phys. Lett. 102B, 263 (1981); Phys. Lett. 115B, 450 (1982).

[5] H. Harari, Sci. Am. 248, 48 (1983).

[6] H. Harari, Phys. Rept. 104, 159 (1984).

[7] M.L. Schmid, A.J. Buchmann, Phys. Rev. D 80, 095020 (2009).

[8] A.J. Buchmann, M.L. Schmid, Phys. Rev. D 71, 055002 (2005).

[9] S. L. Adler, Phys. Lett. B 332, 358 (1994), Nucl. Phys. B415, 195 (1994).

[10] D. Weingarten, Phys. Rev. Lett. 51, 1830 (1983); S. Nussinov, Phys. Rev. Lett. 51, 2081 (1983); E. Witten, Phys. Rev. Lett. 51, 2351 (1983).

[11] J. P. Elliott and P. G. Dawber, Symmetry in Physics (MacMillan, London, 1990), Vol. 2.

[12] To satisfy the 't Hooft anomaly matching conditions, a new group of fermionic rishon bound states, called hyperquarks, was introduced [8]. This led to the condition $N_{G}=N_{C}=N_{H}$, i.e., the equality of the number of generations $N_{G}$, colors $N_{C}$, and hypercolors $N_{H}$. Hyperquarks were later shown to play an important role in explaining the left-right asymmetry of low-energy weak interactions as a residual force on the rishon bound state level [7].

[13] G. 't Hooft, in Recent Developments in Gauge Theories B 59 (Plenum, New York, 1980), p. 135

[14] H. Georgi, Phys. Rev. Lett. 63, 1917 (1989).

[15] L. F. Abott, P. Sikivie, Phys. Lett. 120B, 133 (1983).

[16] Th. Kaluza, Sitzungsber. Pr. Akad. Wiss. Phys. Math. Kl. 54, 966 (1921).

[17] O. Klein, Z. für Physik, 37, 875 (1926); Nature 118, 516 (1926).

[18] S. Nussinov, Phys. Lett. B 165, 55 (1985).

[19] N. Jarosik et al., Astrophys. J. Suppl. 192, 14 (2011).

[20] V. Barger, J. P. Kneller, Hye-Sung Lee, D. Marfatia, G. Steigman, Phys. Lett. B 566, 8 (2003). 